\title{
Can physics principles help explain why non-tuberculous mycobacterial lung disease is more severe in the right middle lobe and lingula?
}

\author{
Sangbong Choi ${ }^{1,2}$, John C. Richards ${ }^{3}$, Edward D. Chan ${ }^{1,4,5}$ \\ ${ }^{1}$ Department of Medicine and Academic Affairs, National Jewish Health, Denver, CO, USA; ${ }^{2}$ Division of Pulmonology and Critical Care Medicine, \\ Inje University Sanggye Paik Hospital, Seoul, South Korea; ${ }^{3}$ Department of Radiology, National Jewish Health, Denver, CO, USA; ${ }^{4}$ Pulmonary \\ Section, Rocky Mountain Regional Veterans Affairs Medical Center, Aurora, CO, USA; ${ }^{5}$ Division of Pulmonary Sciences and Critical Care Medicine, \\ University of Colorado Anschutz Medical Campus, Aurora, CO, USA \\ Correspondence to: Edward D. Chan, MD. D509, Neustadt Building, National Jewish Health, 1400 Jackson St, Denver, CO 80206, USA. \\ Email: chane@njhealth.org.
}

Submitted Mar 22, 2019. Accepted for publication Oct 28, 2019.

doi: $10.21037 /$ jtd.2019.10.70

View this article at: http://dx.doi.org/10.21037/jtd.2019.10.70

\section{Introduction}

Chronic lung disease due to non-tuberculous mycobacteria (NTM) - most often due to organisms belonging to the Mycobacterium avium complex (MAC) - is increasingly prevalent in the United States and many parts of the world. In the non-cystic fibrosis population, NTM lung disease disproportionately affects middle-aged and elderly individuals, and is often recalcitrant to treatment.

NTM lung disease may develop in either pre-existing abnormal lungs, e.g., bronchiectasis or emphysema, or in normal lung tissue. In earlier reports, NTM lung disease was described principally as upper lobe fibrocavitary disease ("classic infection") in patients with underlying emphysema, often mimicking the radiographic pattern of reactivation tuberculosis (Figure 1A) $(1,2)$. This upper lobe pattern is likely secondary to greater frequency of involvement of the upper lobes in smoking-related emphysema, making these areas more vulnerable to NTM infection due to impaired airway clearance, decreased recruitment of immune cells, and reduced lymphatic flow because of the destroyed interstitium (3). However, in the past two to three decades, the nodular-bronchiectasis pattern ("non-classic infection") has been increasingly observed, which may occur in any lobe but appears to be more severe in the right middle lobe (RML) and lingula, and possibly the right upper lobe, typically manifesting as advanced bronchiectasis and atelectasis (Figure 1B,C) $(2,4,5)$.

\section{Evidence for the frequent involvement of the RML and lingula in NTM lung disease}

In a study assessing the CT findings of 24 immunocompromised patients with NTM lung disease (comprised of a total of 143 lobes with the lingula counted as a separate "lobe"), the RML followed by the lingula and the left upper lobe were the most commonly affected by bronchiectasis whereas in 144 lobes in 24 immunocompetent patients with pulmonary NTM, the three lobes most commonly affected by bronchiectasis in descending order of frequency were the right upper lobe, RML, and lingula (Table 1) (6). In another study of 55 patients with lung disease due to MAC, the frequency of bronchiectatic involvement was RML > right upper lobe > lingular segment $>$ right lower lobe $>$ left upper lobe $>$ left lower lobe (7).

The greater severity of NTM lung disease in the RML and lingula is also well supported by the surgical literature. In a retrospective review of 236 consecutive patients who underwent 265 lung resections for NTM lung disease, of the 126 lobectomies, 71 (56\%) were of the RML and of the 55 segmentectomy, 46 (84\%) were of the lingula (Table 1) (9). Despite the fact that the right upper lobe is frequently involved by NTM lung disease $(6,7)$, there were $60 \%$ and $40 \%$ more RML and lingula resections than the right upper lobe, respectively (9), suggesting that disease in the right upper lobe may not be as severe as in the RML and lingula and/or the possibility that surgery of the right upper lobe 

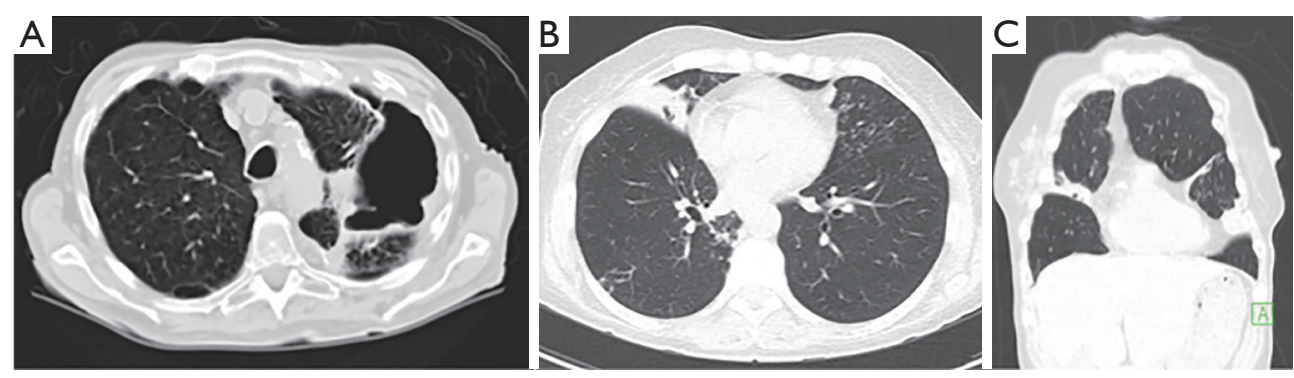

Figure 1 Two principal radiographic patterns for non-tuberculous mycobacteria (NTM) lung disease. (A) Axial CT scan of a "classic infection" pattern with upper lobe fibrocavitary NTM lung disease in an elderly man with underlying emphysema; (B) Axial CT scan of "non-classic" nodular-bronchiectasis pattern with involvement of the right middle lobe (RML) and lingula segment by NTM in a 41-year-old woman; (C) coronal image of same person as in (B) revealing the RML and lingular segment involvement.

Table 1 Differential lobar distribution of NTM lung disease

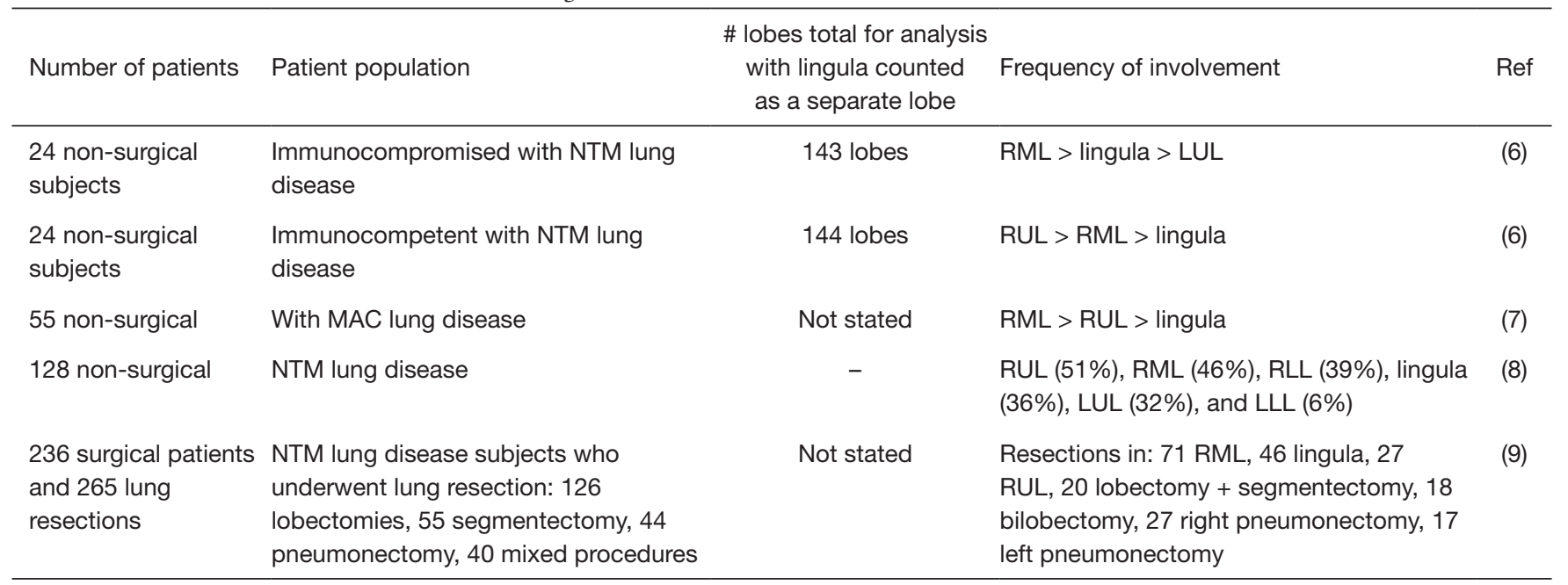

LLL, left lower lobe; LUL, left upper lobe; MAC, Mycobacterium avium complex; NTM, non-tuberculous mycobacteria; RLL, right lower lobe; RML, right middle lobe; RUL, right upper lobe.

may be more technically difficult than either the RML or lingula. However, based on our experience and in discussion with the principal thoracic surgeon who performs nearly all such surgeries at our institution and is the senior author of the aforementioned surgical series (9), the "ease" or lack thereof in surgically removing a lobe plays a minor role in deciding whether to recommend surgery; rather, the clinical factors that collectively play a key role in recommending surgical resection are the presence of severe localized disease, physiologic qualification for undergoing such resections including adequate nutritional status, the absence of prohibitive co-morbid conditions, and patient level of comfort in accepting the risks and benefits. Anecdotally, we have also noticed that after surgical resection of the RML and/or lingula, recurrence of NTM disease may occur in the same area previously occupied by the RML (Figures 2,3) or lingula, suggestive of the possibility that these areas may be more intrinsically more vulnerable to NTM infections. The pattern of which lobes are involved may also be dependent on NTM species and/or the mode of acquisition as an analysis of 14 patients with Mycobacterium chelonae lung disease revealed that bronchiectasis predominantly involved the RML and lingula in only two of the patients and that in the remaining patients, the bronchiectasis was more or less evenly distributed in all the lung lobes (10).

Why the RML and lingula are more severely affected is 


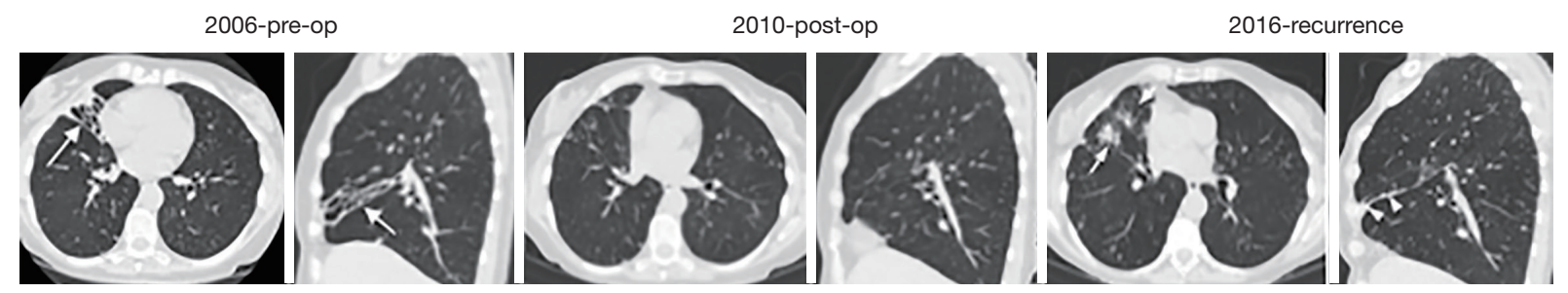

Figure 2 A patient with non-tuberculous mycobacteria (NTM) lung disease due to Mycobacterium avium complex who underwent a right middle lobectomy but many years later, developed recurrence due to Mycobacterium simiae. Pre-operative axial and sagittal CT images from 2006 demonstrated right middle lobe (RML) bronchiectasis and near-complete atelectasis (arrows). Patient underwent right middle lobectomy in 2010 and post-operative images showed minimal abnormality in the lung tissue previously occupied by the RML. In 2016, there was recurrence of NTM lung disease due to $M$. simiae with CT scan showing bronchiectasis and nodularity in the anterior right upper lobe (arrows and arrowheads in the axial and sagittal images, respectively), which now occupied the site of the resected RML.
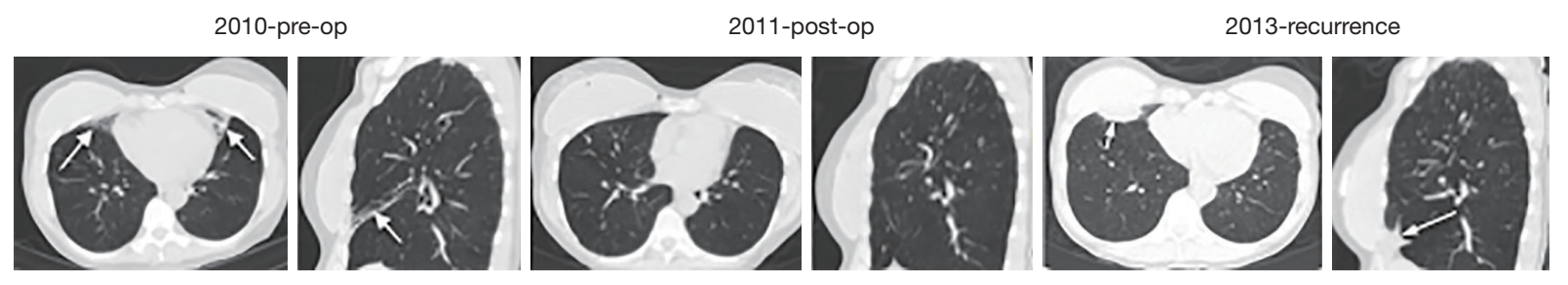

Figure 3 Another patient with non-tuberculous mycobacteria (NTM) lung disease due to Mycobacterium intracellulare who underwent right middle lobectomy and lingulectomy but 2 years later, developed recurrence with Mycobacterium chimaera. Pre-operative axial and sagittal CT images from 2010 showed right middle lobe (RML) and lingular bronchiectasis and atelectasis (arrows) due to M. intracellulare. Patient underwent surgical resection of both the RML and lingula in 2011. Post-operative images showed no abnormalities in the lung tissues previously occupied by the RML and lingula. In 2013, there was recurrence of NTM lung disease with M. chimaera, revealing consolidation in anterior right lower lobe (arrows in both axial and sagittal images), which now occupied the site of the previous RML.

not known. Our goal is to discuss the possible mechanisms for this phenomenon in the context of relevant principles of physics. One obvious criticism for any proposed mechanism is why aren't other non-NTM lung infections necessarily more severe in the RML and lingula as well? While we do not know the answer, we posit that the initial NTM inoculum may have been more or less evenly distributed to both lungs with perhaps a slight predilection for the RML based on mechanisms outlined below. But because of NTM's relatively low virulence, the infections were not suspected to be due to NTM because they were either subclinical or attributed to non-specific "bronchitis". But combined with underlying host risk factor and the presence of recurrent infections-a likely scenario because of the ubiquitous nature of NTM in the environment-the RML and lingular are also less able to clear the infection due to the mechanisms we elaborate below, leading to a chronic infection and more severe disease in those two areas. Moreover, it is reasonable to posit that in someone with a "routine" bacterial respiratory infection, empiric therapy with antibiotics are likely to be effective, perhaps accounting for lack of preferential involvement of the RML and lingula segment with acute non-NTM infections. But the empiric antibiotic used and the length of treatment may be inadequate for NTM infection, which usually requires more specific, multiple antibiotics and a much longer duration of treatment. Thus, short course, mono-antibiotic therapy of an unsuspected NTM infection may allow the disease process to fester and develop into an established chronic infection with few alarming signs and symptoms until advanced. 

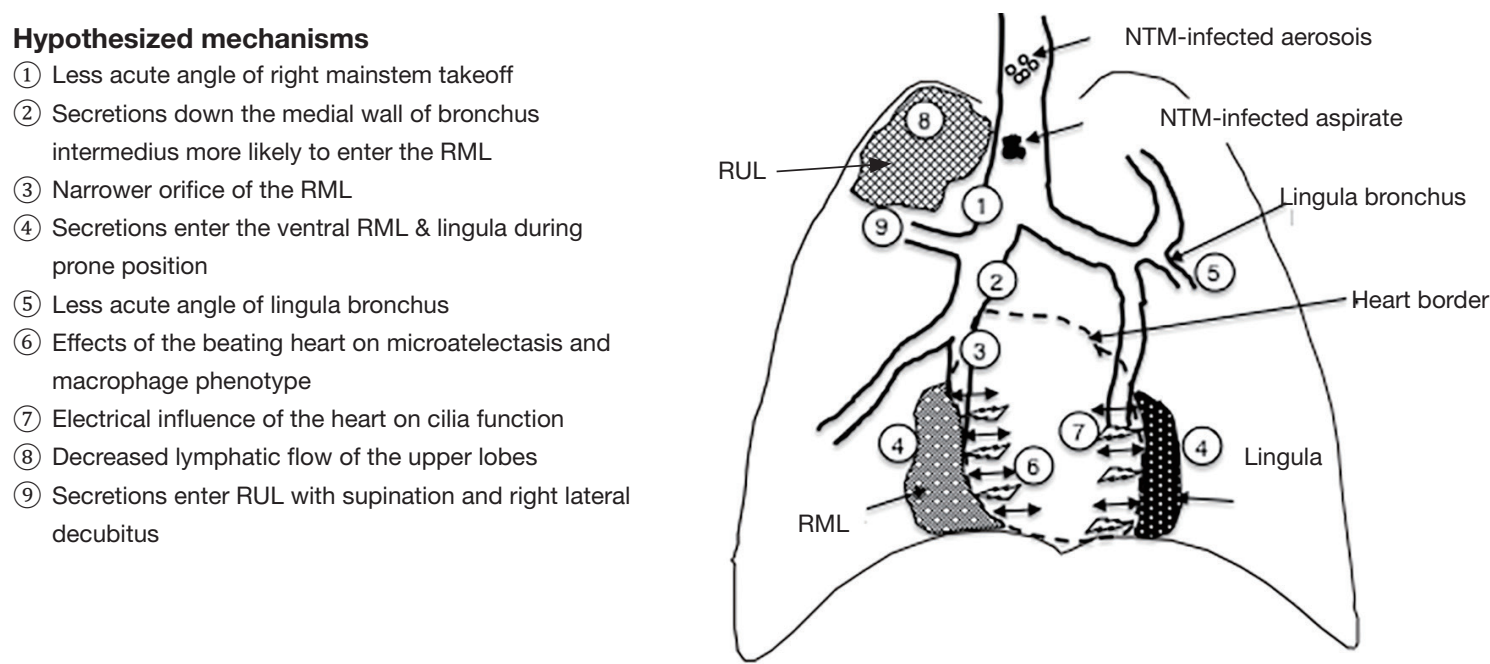

Figure 4 Cartoon of the hypothesized mechanisms by which the right middle lobe and lingula, and to a lesser extent the right upper lobe are more severely affected by NTM lung disease. Please also see text for discussion. NTM, non-tuberculous mycobacteria; RML, right middle lobe; RUL, right upper lobe.

\section{Hypothesized mechanisms to explain greater frequency and/or severity of NTM lung disease involving the RML and lingula}

\section{Gravitational and fuid dynamic influences}

The two major modes of acquisition of isolated NTM lung disease are (I) inhalation of NTM-contaminated water, biofilm or soil aerosols and (II) microaspiration, which may be from "above" due to swallowing dysfunction and/or from "below" due to high gastroesophageal reflux (Figure 4) (11).

While microaspiration may occur in any segment or lobe of the lungs_-depending on the volume of the aspirate and the position/posture of the subject at the time of the aspiration(s) - there are several "truisms" of gravitational and fluid dynamic principles that should be considered.

First, because in adults the right mainstem bronchus makes a less acute angle at its takeoff from the trachea (i.e., the right mainstem bronchus is more vertical in the upright position) than the left mainstem bronchus (which is less vertical), it stands to reason that any inhaled aerosol or aspirate is more likely to enter the right lung than the left lung. In addition, the right mainstem bronchus is shorter than the left mainstem bronchus - an average of 2.5 vs. $5 \mathrm{~cm}$, respectively_as well as possessing a slightly wider transverse diameter (12). This paradigm is supported by the clinical experience that foreign body aspiration in adults is more likely to lodge in the right lung, occurring in $60-70 \%$ of cases compared to $30-40 \%$ for the left lung in two studies $(13,14)$. In contrast, foreign body aspiration in children occur roughly equally in the left and right lungs because of approximate equal size and angulation of their mainstem bronchi (15). This potential increased susceptibility of the right lung in adults to aspiration based on this anatomic finding is supported by the finding that in 128 patients with NTM lung disease, bronchiectasis more commonly affected the lobes of the right lung than the left (\% involved): right upper lobe (51\%), RML (46\%), right lower lobe (39\%), lingula (36\%), left upper lobe (32\%), and left lower lobe (6\%) (Table 1) (8). The greater involvement of the individual lobes of the right lung is also mirrored by the surgical literature in which right pneumonectomy was performed in 27 patients and left pneumonectomy in 17 patients in a series of 236 patients who underwent lung resections for NTM lung disease (9).

Second, because of the hyperacute angle takeoff of both of the right and left upper lobe bronchi-typically greater than $90^{\circ}$ using the longitudinal trachea as the reference plane-aspiration to the upper lobes would appear less likely when one takes into account gravitational forces (if person is upright) or air/fluid flow dynamics influenced by directional momentum; i.e., flow maintains its original direction unless impacted by external forces. An example of the latter is that of a river that divides into several tributaries; i.e., from the parent river, water is more likely to flow into a tributary 
that forms a less acute angle than one that forms a more acute angle with other factors such as gradients being equal. However, aspiration may obviously occur in any part of the lungs if the aspirate volume is relatively large or depending on the position/posture of the patient at the time of the aspiration. In light of this, the relative frequent involvement of the right upper lobe with NTM lung disease may be related to several factors, including: (I) greater propensity of proximal aspirates to initially enter the right lung in the first place, (II) secretions traveling down the lateral wall of the right mainstem bronchus are likely to enter the orifice of the right upper lobe bronchus-especially when lying down-due to the adhesive properties of water molecules to each other, (III) right lateral decubitus positioning during sleep, and (IV) decreased lymphatic flow to the upper lobes (3).

Third, because the lateral wall of the right mainstem bronchus is interrupted by the orifice of the right upper lobe bronchus, it is likely that secretions traveling down the bronchus intermedius are more likely to flow along the medial wall than the lateral wall, preferentially entering the more medial RML bronchus than the more lateral right lower lobe bronchus. However, depending on the aspirate volume and the position of the patient at the time of the aspiration, the right lower lobe may also be affected. Indeed, in the aforementioned study of 128 patients with NTM lung disease, the right lower lobe was six times more commonly involved compared to the left lower lobe, likely reflecting the preferential involvement of the right lung with NTMinfected aspirate or inhaled aerosols (8). Moreover, if the inoculum size is important for establishment of an infection, it seems plausible that the density of the inoculum in the smaller RML would be greater than that of the larger right lower lobe, where there are multiple segments to distribute the inhaled or aspirated NTM and thus could account for greater disease severity in the RML.

Fourth, the lingula is technically part of the left upper lobe but its bronchial segment takes off the left mainstem bronchus at a less acute angle than the left upper lobe proper and thus may be a preferential site of pooling by aspirated secretions that travel down the left lung (Figure 4).

Fifth, if patients slept in the prone position, one could reasonably posit that any occult aspiration during sleep may be more likely to occur repeatedly in the ventrally-located RML and lingula. Because there is less time spent in the prone position with aging $(16,17)$ and NTM lung disease is more likely to occur in the elderly, it would seem that prone position is a less tenable mechanism to explain the more severe disease in the RML and lingula. On the other hand, it is plausible that those with more severe disease in the RML and lingula may in fact be the minority of older individuals who prefer to sleep prone.

\section{Mechanical/anatomical influences}

Could intrinsic anatomic factors account for the greater severity of NTM lung disease in the RML and lingula? The orifice of the RML bronchus is more narrowed than other bronchi, a well-known anatomic landmark often noted to resemble a "fish mouth" by bronchoscopists. Thus, it seems plausible that during forced exhalation as seen with a cough, especially in the presence of collapsible airway walls, the RML bronchus may be handicapped in clearing out any distal secretions. Byrd and co-workers (18) posited that the long and narrow bronchi of the RML and lingula may predispose these lung segments to retain secretions more easily.

Lee and co-workers (19) compared the lengths, diameters, and angles of the RML and lingular bronchi of 100 healthy subjects with normal chest CT with that of 50 individuals with NTM lung disease that involved the RML and lingular segment matched for gender, age, height, and body weight. Using multiplanar reformation images to make their measurements, they found no difference in the lengths or diameters of the RML or lingular bronchi between the two groups but instead found a significant difference in the takeoff angles of the RML and lingular bronchi between NTM lung disease subjects and healthy controls. The angulations of the RML and lingular segment bronchi were defined - on an axial CT image-as the angle that forms between a coronal plane with the plane of the RML bronchus and lingular bronchus, respectively. More specifically, they found that the RML and lingular bronchi angles were significantly greater in healthy subjects (mean $52^{\circ} \pm 8^{\circ}$ and $35^{\circ} \pm 10^{\circ}$, respectively) than those of NTM lung disease subjects (mean $47^{\circ} \pm 9^{\circ}$ and $27^{\circ} \pm 8^{\circ}$, respectively); i.e., the RML and lingular bronchi make a greater turnmore acute angle-in the healthy subjects than in patients with NTM lung disease. While these measurements were not available for the pulmonary NTM subjects before they developed their lung disease, it would seem that any atelectasis of the RML and lingula would cause a greater acuity of their bronchial takeoff; i.e., their finding of a less acute angle of the RML and lingula bronchi in the NTM lung disease subjects would lend credence that pre-existing anatomical differences may indeed play a role in why these 
two areas of the lungs are more severely affected with the nodular-bronchiectasis form of NTM lung disease.

Another potential anatomic factor for the greater RML and lingular severity in NTM lung disease is that both these two areas about the beating heart. It seems plausible that the constant rhythmic compression of the heart on the adjacent RML and lingula may cause microatelectasis in the lung regions closest to the heart and, in the presence of other risk factors for NTM lung disease, sets up a nidus of sustained infection that then spreads to the other parts of the RML and lingula. While we have no evidence for this occurring, one of the mechanisms by which prone positioning a patient with acute respiratory distress syndrome helps improve oxygenation is that it alleviates the heart, located ventrally, from compressing the dorsal lung (20). An additional possibility is that any such cardiac "tapping" of the adjacent lungs may result in skewing of macrophages in those areas to an alternative phenotype ("wound healing macrophages") that are impaired in host defense against NTM, based on the phenomenon of locus minoris resistentiae ("site of least resistance"); i.e., tissues with prior or current trauma are more prone to infections (21). However, unless there are other risk factors involved, the cardiac tapping-induced microatelectasis and influence on macrophage phenotype seems untenable as the only purported mechanisms since these factors would also be present in the general population, the vast majority of whom do not have NTM lung disease. Nevertheless, the anecdotal observation by some that the RML is often more severely involved compared to the lingula in NTM lung disease is likely due to: (I) the greater risk of aspiration to the right lung, (II) the narrower orifice of the RML bronchus, and (III) the much less acute angle by which the RML bronchus takes off from the bronchus intermedius compared to the more acute angle of the takeoff of the lingula bronchus from the left mainstem bronchus.

\section{Electrical influences}

Patients with primary ciliary dyskinesia are predisposed to bronchiectasis and NTM lung disease although it is not known with certainty whether the NTM lung disease is a sequela of pre-existing bronchiectasis or the bronchiectasis is the result of unresolved NTM lung infection; both scenarios likely occur as bronchiectasis from other causes-resulting in secondary ciliary dysfunction-is also a predisposing factor for NTM lung disease. Fowler et al. found reduced ciliary beat frequency in the nasal scrapings of NTM lung disease subjects compared to healthy controls (22). While this finding would suggest a more widespread ciliary defect-i.e., ciliary defect not just isolated to the lungs-we query whether it is possible that the electrical activity of the heart may reduce the ciliary beat frequency or coordination of their beats in the RML and lingula tissues that abut the heart?

There is no doubt that cardiac electrical activity can be transmitted to a site relatively far distant from the heart as evinced by the body surface electrocardiogram. We thus hypothesize that the ciliated airways of the RML and lingula, being closest to the heart, may possibly be more easily perturbed by cardiac electrical activity. Supporting this hypothesis is that in addition to the canonical cells and fibers that conduct electrical signals in the heart, macrophages in the myocardium were found to be coupled to spontaneously beating cardiac myocytes also via connexin 43 and that the macrophages depolarize in synchrony with the myocytes, resulting in more coordinated contraction of the myocardium $(23,24)$. Since cardiac tissue macrophages are in close contact with the epicardium and are known to affect the activity of the multi-potent progenitor cells located in the epicardium, it is interesting to speculate that myocardial macrophages may also transmit electrical signals to the pericardium. The pericardium is comprised of the outer, fibrous parietal layer and the inner visceral layer with the latter comprised of an outer layer of mesothelial cells and an inner loose fatty connective tissue layer that contain the coronaries, nerves, and macrophages. Because abnormalities of the pericardium can also be detected by surface electrocardiogram, one wonders whether any electrical activity of the heart that is transmitted through the pericardium could potentially alter the function of lung macrophages and/or airway cilia in the regions of the RML and lingular segment that abut the heart?

Moreover, sessile lung macrophages are known to dampen lipopolysaccharide-induced inflammation by directly communicating with lung epithelial cells also via connexin 43 -containing gap junction channels, causing calciumdependent Akt activation in alveolar epithelial cells, and subsequently reducing both pro-inflammatory cytokine production and neutrophil recruitment in the lungs (25). In contrast to this anti-inflammatory effect of connexin 43 , others have shown in a sepsis model that macrophage connexin 43 is upregulated in sepsis, resulting in increased pro-inflammatory cytokine expression and reduced survival in mice (26). Despite this seeming controversy, the role connexin 43 may play with chronic infections such as 
NTM lung disease remains to be determined with two possible and not necessarily mutually exclusive conjectures in the context of the RML and lingula paradigm: an early immunosuppressive effect of connexin 43 may allow NTM infection to persist whereas later pro-inflammatory effects of connexin 43 may result in lung tissue injury. In addition, since NTM lung disease is airway-centric and cilia essentially line the entire airway luminal wall, any potential adverse perturbation of ciliary function from the adjacent heart through the connexin 43-containing gap junction channels - present in the heart, the lung epithelium, and the macrophages in both organs-would be expected to facilitate sustained infections of both smaller and larger airways.

This concept of electrical activity affecting macrophage function is supported by studies showing that ion channel mediated electrical conductance may alter the ability of macrophages to respond to extracellular signals that mediate chemotaxis and phagocytosis (27). But unless there are other host risk factors and significant environmental exposure, the possibility that electrical activity of the heart altering ciliary function in the lungs or lung macrophage activation as the only risk factor for greater predisposition of the RML and lingula to NTM lung disease seems implausible since this hypothesized pathophysiology would also be present in the general population.

\section{Conclusions}

In conclusion, we posit that several factors-including gravitational/fluid dynamic influences, mechanical/ anatomical influences, and electrical influences-in the background of underlying host and environmental risks, predispose the RML and lingular segment to chronic infection with NTM in susceptible individuals, resulting in more severe disease in these areas (Figure 4). We did not discuss the role of the microbes or microbe-host interactions in influencing the preferred sites of infection. However, it is a reasonable supposition that NTM-mediated biofilmsmost likely formed from a combination of both NTMderived and host-derived extracellular biomoleculessynergize with the aforementioned physical mechanisms to allow greater persistence of the NTM in those areas of the lungs that are likely to be initially colonized, causing airway and parenchymal lung destruction.

\section{Acknowledgments}

We are grateful to Richard K. Albert, M.D. for critical read of the manuscript and helpful suggestions.

\section{Footnote}

Conflicts of Interest: The authors have no conflicts of interest to declare.

Ethical Statement: The authors are accountable for all aspects of the work in ensuring that questions related to the accuracy or integrity of any part of the work are appropriately investigated and resolved.

\section{References}

1. Christensen EE, Dietz GW, Ahn CH, et al. Pulmonary manifestations of Mycobacterium intracellularis. AJR Am J Roentgenol 1979;133:59-66.

2. Erasmus JJ, McAdams HP, Farrell MA, et al. Pulmonary nontuberculous mycobacterial infection: radiologic manifestations. Radiographics 1999;19:1487-505.

3. Gurney JW, Schroeder BA. Upper lobe lung disease: physiologic correlates. Radiology 1988;167:359-66.

4. Hartman TE, Swensen SJ, Williams DE. Mycobacterium avium-intracellulare complex: evaluation with CT. Radiology 1993;187:23-6.

5. Miller WT Jr. Spectrum of pulmonary nontuberculous mycobacterial infection. Radiology 1994;191:343-50.

6. Lee Y, Song JW, Chae EJ, et al. CT findings of pulmonary non-tuberculous mycobacterial infection in non-

AIDS immunocompromised patients: a case-controlled comparison with immunocompetent patients. Br J Radiol 2013;86:20120209.

7. Lynch DA, Simone PM, Fox MA, et al. CT features of pulmonary Mycobacterium avium complex infection. J Comput Assist Tomogr 1995;19:353-60.

8. Mitchell JD, Bishop A, Cafaro A, et al. Anatomic lung resection for nontuberculous mycobacterial disease. Ann Thorac Surg 2008;85:1887-92.

9. Hazelton TR, Newell JD Jr, Cook JL, et al. CT findings in 14 patients with Mycobacterium chelonae pulmonary infection. AJR Am J Roentgenol 2000;175:413-6.

10. Koh WJ, Lee JH, Kwon YS, et al. Prevalence of gastroesophageal reflux disease in patients with nontuberculous mycobacterial lung disease. Chest 2007;131:1825-30.

11. Muresian H. Pleura, lungs, trachea and bronchi. In: Standring S. editor. Gray's Anatomy: The Anatomical Basis of Clinical Practice, 41st ed. Elsevier; 2015, 953-69. 
12. Baharloo F, Veyckemans F, Francis C, et al. Tracheobronchial foreign bodies: presentation and management in children and adults. Chest 1999; 115:1357-62.

13. Sehgal IS, Dhooria S, Ram B, et al. Foreign body inhalation in the adult population: Experience of 25,998 bronchoscopies and systematic review of the literature. Respir Care 2015;60:1438-48.

14. Feller-Kopman D, Mehta AC, Wahidi MM. Therapeutic bronchoscopy. In: Broaddus VC, Mason RJ, Ernst JD, et al. editors. Murray \& Nadel's Textbook of Respiratory Medicine, 6th ed. Philadelphia, PA: Saunders; 2016, 383-92.

15. Kim C, Park SH, Oh SY, et al. Comparison of chest CT findings in $\mathrm{n}$ ontuberculous mycobacterial diseases vs. Mycobacterium tuberculosis lung disease in HIV-negative patients with cavities. PLoS One 2017;12:e0174240.

16. De Koninck J, Lorrain D, Gagnon P. Sleep positions and position shifts in five age groups: an ontogenetic picture. Sleep 1992;15:143-9.

17. Lorrain D, De Koninck J, Dionne H, et al. Sleep positions and postural shifts in elderly persons. Percept Mot Skills 1986;63:352-4.

18. Byrd R Jr, Payne JL, Roy TM. Lingular and middle lobe infiltrates in an elderly woman. Chest 1995;108:1156-7.

19. Lee T, Park JY, Lee HY, et al. Bronchial angles are associated with nodular bronchiectatic non-tuberculous mycobacteria lung disease. Int J Tuberc Lung Dis

Cite this article as: Choi S, Richards JC, Chan ED. Can physics principles help explain why non-tuberculous mycobacterial lung disease is more severe in the right middle lobe and lingula? J Thorac Dis 2019;11(11):4847-4854. doi: $10.21037 /$ jtd.2019.10.70
2017;21:1169-75.

20. Benson AB, Albert RK. Prone positioning for acute respiratory distress syndrome. Clin Chest Med 2014;35:743-52.

21. Chan ED, Kong PM, Fennelly K, et al. Vertebral osteomyelitis due to infection with nontuberculous Mycobacterium species after blunt trauma to the back: 3 examples of the principle of locus minoris resistentiae. Clin Infect Dis 2001;32:1506-10.

22. Fowler CJ, Olivier KN, Leung JM, et al. Abnormal nasal nitric oxide production, ciliary beat frequency, and Tolllike receptor response in pulmonary nontuberculous mycobacterial disease epithelium. Am J Respir Crit Care Med 2013;187:1374-81.

23. Hulsmans M, Clauss S, Xiao L, et al. Macrophages facilitate electrical conduction in the heart. Cell 2017;169:510-522.e20.

24. Rosenthal N. A guardian of the heartbeat. N Engl J Med 2017;377:84-6.

25. Westphalen K, Gusarova GA, Islam MN, et al. Sessile alveolar macrophages communicate with alveolar epithelium to modulate immunity. Nature 2014;506:503-6.

26. Dosch M, Zindel J, Jebbawi F, et al. Connexin-43dependent ATP release mediates macrophage activation during sepsis. Elife 2019;8:e42670.

27. Nelson DJ, Jow B, Popovich KJ. Whole-cell currents in macrophages: II. Alveolar macrophages. J Membr Biol 1990;117:45-55. 